\title{
Labyrinthes dans le désert. Deux pèlerinages dans le nord-ouest mexicain
}

\section{Neyra Alvarado Solís}

\section{OpenEdition}

12 Journals

Édition électronique

URL : https://journals.openedition.org/jsa/11376

DOI : 10.4000/jsa. 11376

ISSN : 1957-7842

\section{Éditeur}

Société des américanistes

\section{Édition imprimée}

Date de publication : 5 juin 2010

Pagination : 193-213

ISSN : 0037-9174

\section{Référence électronique}

Neyra Alvarado Solís, "Labyrinthes dans le désert. Deux pèlerinages dans le nord-ouest mexicain », Journal de la Société des américanistes [En ligne], 96-1 | 2010, mis en ligne le 10 décembre 2014, consulté le 03 septembre 2022. URL : http://journals.openedition.org/jsa/11376 ; DOI : https:// doi.org/10.4000/jsa. 11376 


\title{
LABYRINTHES DANS LE DÉSERT. DEUX PÈLERINAGES DANS LE NORD-OUEST MEXICAIN
}

\author{
Neyra ALVARADO SOLÍS *
}

Les pèlerinages à pied dans le désert mexicain illustrent des façons de s'approprier l'espace qui se distinguent de celles de la tradition mésoaméricaine, pour laquelle il existe une cartographie stable du paysage parcouru sur des chemins qui ne changent guère. On voit cela chez les Papago du Sonora (Mexique) et de l'Arizona (États-Unis) lors de leurs pèlerinages au sanctuaire de saint François-Xavier à Magdalena de Kino et chez les pèlerins métis de Villa de Cos, Zacatecas, qui visitent l'image sainte de saint François à Real de Catorce, San Luis Potosi. Pendant le trajet, les deux pèlerinages associent des pratiques rituelles à des repères constitués par des lieux, des cactus et des animaux considérés comme dangereux. Aucune identification de ces endroits n'est donnée à l'avance : ils sont reconnus au moment où les pèlerins les trouvent. Ces façons de s'approprier l'espace mettent en scène les désordres du corps et l'image sainte, tous deux associés à la mémoire et au dispositif du savoir, ce qui permet une réflexion sur la conception des ancêtres et l'image du labyrinthe. [Mots-clés: Mexique, pèlerinage, espace, désert, corps, image sainte.]

Laberintos en el desierto. Dos peregrinaciones en el Noroeste mexicano. Las peregrinaciones a pie, en el desierto mexicano, permiten conocer otra forma de apropiarse el espacio, en contraste con las peregrinaciones de tradición mesoamericana, donde existe una cartografia estable del paisaje y cuyos caminos casi no cambian. Lo vemos entre los pápagos de Sonora, México, y los de Arizona, Estados Unidos, quienes van en peregrinación hacia el santuario de San Francisco Xavier en Magdalena de Kino y entre los peregrinos mestizos de Villa de Cos, Zacatecas, quienes visitan la imagen santa de San Francisco en Real de Catorce, San Luis Potosí. Las dos peregrinaciones asocian unas prácticas rituales, durante la marcha, a las marcas de lugares, cactus y animales concebidos como peligrosos. Ninguna identificación de esos lugares se hace previamente, éstos son reconocidos al cruce de los peregrinos. Estas formas de apropiarse el espacio así como los ritos ponen en escena el cuerpo en crisis y la imagen santa, ambos asociados a la memoria y al dispositivo del saber, lo que nos lleva a reflexionar sobre los ancestros y la noción de laberinto. [Palabras claves : México, peregrinación, espacio, desierto, cuerpo humano, imagen santa.]

* El Colegio de San Luis A. C., Parque Macul 155, Fraccionamiento Colinas del Parque, CP 78299, San Luis Potosi, SLP, Mexique [nalvarado@colsan.edu.mx].

Journal de la Société des Américanistes, 2010, 96-1, pp. 193-213. C Société des Américanistes. 
Labyrinths in the desert. Two pilgrimages in the Northwest of Mexico. Walking pilgrimages through the Mexican desert allow another way to apprehend space. These differe in essence from the traditional Mesoamerican pilgrimages, which have a landscape cartography with defined paths that almost never change. The Papago tribes of Sonora, Mexico, and Arizona, USA, make a walking pilgrimage to the Sanctuary of San Francisco Javier at Magdalena de Kino and the Mestizo pilgrims from Villa de Cos in Zacatecas visit the sacred image of San Francisco at Real de Catorce, San Luis Potosi. Both pilgrimages include rituals along the route associated to places, cactus and animals thought of as dangerous. No identification of these places sites is previously defined : they are fortuitously recognized by the pilgrims as they discover them, along the walk. These ways to apprehend the space and the rituals, elevate the human body to a special state of being, along with the image of the Saint, associated to the memory and knowledge about ancestors, which allow us to think about them and the notion of a labyrinth. [Key words : Mexico, pilgrimage, space, desert, human body, sacred image.]

\section{INTRODUCTION}

Le désert mexicain, connu sous les noms de chihuahuense et de sonorense ${ }^{1}$, est balisé par des sanctuaires visités régulièrement tant par les populations indiennes que métisses. Nombre de pèlerinages se font en autobus et quelques-uns suivent le trajet d'un voyage touristique. Bien différentes sont les processions à pied ${ }^{2}$, lesquelles mettent en avant le corps souffrant et le sacrifice corporel. Après avoir accompli huit de ces pèlerinages, il nous est apparu que ce type de déplacement entretient une relation étroite avec l'environnement. Néanmoins, ce qui peut sembler paradoxal est qu'il n'existe pas, dans l'imaginaire des acteurs, de véritable cartographie rituelle des paysages traversés.

Dans ces conditions, comment les pèlerins construisent-ils des catégories ad hoc d'" espace » et de " temps » ? Comment le corps contribue-t-il à déclencher un dispositif mnémonique spatial, voire temporel ? Nous chercherons des éléments de réponse en comparant deux pèlerinages. Le premier est celui des Indiens papago (Tohono O'otham) de Sonora et de l'Arizona, le second celui des métis de Villa de Cos, dans l'État de Zacatecas. Les Papago se dirigent de Tubutama vers le sanctuaire consacré à l'image sainte de saint François-Xavier, à Magdalena de Kino, Sonora. Les métis partent de Sierra Hermosa vers le sanctuaire de saint François situé à Real de Catorce, San Luis Potosi. Pendant cinq jours, pour l'un, et six jours, pour l'autre, ils parcourent respectivement cinquante et quatre-vingts kilomètres.

Les enquêtes de terrain chez les Papago de l'Arizona sont difficiles dans la mesure où les autorités de la réserve de Sells interdisent les recherches sur leur territoire. Toutefois, il m'a été possible d'observer ce pèlerinage dans le désert de l'État de Sonora en 2003 après avoir fait en 2002 d'autres séjours chez les Papago 
des deux côtés de la frontière internationale. En ce qui concerne la population métisse de Villa de Cos, Zacatecas, aucune enquête n'avait été effectuée jusqu'à présent. Les données présentées ici sont donc originales, s'agissant d'un domaine méconnu dans l'ethnographie du Mexique. Les recherches sur les pèlerinages dans le désert mexicain sont au demeurant rares. On trouve des mentions du pèlerinage papago dans quelques travaux, notamment dans une étude générale sur les Mexicano-Américains (Howart et Lamadrid 1999) qui rendent visite au Seigneur d'Esquipulas à Chimayo, Nouveau Mexique, rite qui montre la perméabilité entre catholicisme et religion tewa. Cramaussel et Álvarez (1994), de leur côté, ont pu établir que la confrérie des Morados, organisation religieuse du Señor de los Guerreros "seigneur des guerriers ", s'étendait aux XVI ${ }^{e}$ et XVII siècles sur l'ancienne province de Santa Barbara, bien qu'à présent l'organisation ne coïncide pas complètement avec la région. Reyna (2005) a étudié, pour sa part, le pèlerinage de la Milice de San Miguel dont le nom exprime une organisation religieuse, de type militaire, extrêmement complexe. Quant à Del Moral González (2006), elle a pu mettre en lumière comment, dans le pèlerinage vers le sanctuaire du Señor de Mapimí, l'image sainte est investie de caractéristiques d'origine indienne en liaison avec le paysage et les pluies dans un rite pourtant métis. Cependant l'analyse spatiale, notamment du désert, est absente dans toutes ces recherches.

Les pèlerinages les mieux connus du Mexique concernent le centre et le sud du pays et privilégient le point de vue du sanctuaire, sans que la question de la représentation de l'espace par les pèlerins soit abordée. Certaines études soulignent les racines indiennes de ces manifestations, en particulier pour les sanctuaires d'origine préhispanique, comme celui de Tila au Chiapas, où l'on vénère une image préhispanique maya associée au Christ (Monroy 1995), ou bien le sanctuaire de Chalma érigé sur un lieu de culte préhispanique (Shadow et Rodríguez Shadow 2000). Une seule recherche collective sur le sanctuaire d'Otatitlan et les pèlerinages régionaux qui s'y déroulent met en exergue l'existence d'une « aire dévotionnelle » aux frontières discontinues (Velasco 1997, pp. 32-34). On y voit comment l'espace est ponctué de points de repère, là où se trouvent des sources ou des arbres liés à des entités solaires ou lunaires évoquant le premier couple cosmogonique. Les Huichol empruntent, eux aussi, chaque année un trajet marqué par des lieux précis, visités avant d'atteindre l'endroit où réside le peyotl-soleil ; pour eux, le désert est reunari, un des pôles de l'univers, celui de la sortie du soleil (Gutiérrez 2002).

Les quelques recherches disponibles restituent une conception de l'espace de tradition mésoaméricaine, selon laquelle certains endroits associés aux mythes de création et aux divinités cosmogoniques doivent être régulièrement visités. La disposition spatiale qu'on y découvre est marquée par le chiffre cinq, chiffre de la complétude du monde humain. On trouve aussi quelques références au corps soumis au sacrifice corporel, peu ou pas analysées. 
Dans les déserts chihuahuense et sonorense, la représentation rituelle de l'espace par les pèlerins papago et métis relève d'une autre démarche. Le désert est un espace en apparence vide, mais où le corps a une place déterminante dans sa construction et dans la mémoire. En fait, il semble bien qu'il existe une différence conceptuelle de l'espace entre les cultures de tradition mésoaméricaine et celles du nord du Mexique. Dans les pages qui suivent, on résumera le déroulement des deux pèlerinages étudiés en se référant au culte des images saintes qui déterminent le mouvement des fidèles, mais également à l'organisation religieuse propre au voyage et à la marche à travers le désert.

\section{ANALySes}

\section{Les images saintes, les origines}

Les deux pèlerinages ont une origine récente. Celui des Papago a débuté en 1997, à la suite du vœu d'un chamane, aujourd'hui responsable du pèlerinage, de devenir abstème ${ }^{3}$. Celui des métis remonte à 1960 et a vu le jour au sein d'une population fortement marquée par les croyances en la force de saint François dès l'évangélisation franciscaine dans la région (Figure 1).

\begin{tabular}{|l|c|c|c|c|c|c|}
\hline \multicolumn{1}{|c|}{ Pèlerinage } & $\begin{array}{c}\text { Date d'origine } \\
\text { et motivation }\end{array}$ & Religion & $\begin{array}{l}\text { Image sainte } \\
\text { du sanctuaire }\end{array}$ & Sexe & Parenté & $\begin{array}{l}\text { Saint éponyme } \\
\text { du lieu saint }\end{array}$ \\
\hline $\begin{array}{l}\text { De Villa de } \\
\text { Cos, Zacatecas } \\
\text { à Real de Ca- } \\
\text { torce, San Luis } \\
\text { Potosi }\end{array}$ & $\begin{array}{c}1960 \\
\text { Foi }\end{array}$ & Catholique & $\begin{array}{l}\text { saint François } \\
\text { d'Assise } \\
\text { (franciscain) }\end{array}$ & Masculin & Frère & $\begin{array}{l}\text { Vierge de la } \\
\text { Conception }\end{array}$ \\
\hline $\begin{array}{l}\text { Tubutama vers } \\
\text { Magdalena de } \\
\text { Kino, Sonora }\end{array}$ & $\begin{array}{l}1997 \\
\text { Vouu }\end{array}$ & $\begin{array}{l}\text { Catholique } \\
\text { indianisée }\end{array}$ & $\begin{array}{l}\text { saint François- } \\
\text { Xavier } \\
\text { (jésuite) }\end{array}$ & Masculin & Frère & $\begin{array}{l}\text { Vierge Marie- } \\
\text { Madeleine }\end{array}$ \\
\hline
\end{tabular}

FIG. 1 - Images saintes dans les pèlerinages étudiés.

Dans les deux cas, les pèlerins insistent sur la puissance des images, attestée par des miracles répétés au fil du temps. La puissance "ancienne » attribuée aux images saintes ne cadre pas avec le caractère récent des pèlerinages, ladite puissance étant conçue comme ayant toujours existé. Cette contradiction peut être résolue si l'on situe ces qualités des images saintes dans une conception cyclique du temps.

Chacune des images se révèle historiquement importante pour les régions où habitent les pèlerins. L'image de saint François-Xavier arrive dans la Papaguería - nom donné à la région où habitaient les Papago - avec les missionnaires jésuites à l'époque coloniale (Alvarado 2007). Aujourd'hui, chez les Indiens et chez 
les métis, on le commémore à la même date que celle de la fête du fondateur des franciscains, saint François d'Assise, ce qui tient peut-être à la présence et à l'influence de groupes de missionnaires au sein de cette population. À Magdalena de Kino, le sanctuaire contient des images de saint FrançoisXavier, tandis que d'autres sont conservées dans les communautés et sur des autels domestiques à l'intérieur des maisons. Le nom du sanctuaire associe Marie-Madeleine, la sainte, et Kino, le nom du célèbre jésuite qui a évangélisé la région et dont les reliques se trouvent dans une tombe au cœur du sanctuaire. Saint François-Xavier est par ailleurs un saint miraculeux, qui possède les caractéristiques marines d'une ogresse anthropophage et celles du héros culturel I'toi, tous les deux ayant participé à la création du monde papago (Galinier 1991, pp. 534-535). Il faut garder à l'esprit les caractéristiques attribuées à l'image sainte de saint François-Xavier en relation avec le monde papago des origines et la façon dont les Papago ont intégré des dévotions catholiques dans leur cosmogonie.

L'image de saint François est arrivée à Real de Catorce avec les missionnaires franciscains, mais c'est à la fin du $\mathrm{XIX}^{\mathrm{e}}$ siècle, au terme de l'essor minier, et plus encore au $\mathrm{xx}^{\mathrm{e}}$ siècle qu'elle commence à attirer les pèlerins. Real de Catorce fut un centre minier dont le saint éponyme originel fut la Vierge de la Conception, mais l'action évangélisatrice des franciscains dans la région a fait de saint François un personnage particulièrement miraculeux. Ainsi, pour les pèlerins, son image est censée les aider à jouir d'une meilleure vie sur terre et après la mort.

Papago et métis accordent une place importante à saint François, le jésuite et le franciscain respectivement, et ce en dépit de l'origine récente des pèlerinages liée, avons-nous déjà dit, à un acte de foi des pèlerins, dans le cas des métis, et à un vœu du chamane pour obtenir sa guérison, dans le cas indigène.

Autant chez les Indiens que chez les métis, ces deux images saintes sont conçues par les pèlerins comme des intercesseurs devant Dieu et, de plus, comme deux frères, fils de ce dernier et de la Vierge. Leur aide est fondamentale pour obtenir une longue vie, vœu qui peut se réaliser grâce au voyage rituel et à son organisation religieuse.

\section{Les organisations religieuses}

Les deux pèlerinages requièrent une organisation religieuse pour répondre aux besoins et aux contraintes de la marche dans le désert (Figure 2). Les pèlerins métis sont des employés de commerce, des femmes de ménage ou des fonctionnaires, tandis que, chez les Papago, le groupe est composé d'étudiants, de femmes au foyer ou de fonctionnaires. Tous demandent des congés pour y participer. Chaque année, ces pèlerinages attirent de nouveaux fidèles, ainsi qu'en témoignent, chez les métis, l'existence de rituels d'intégration ${ }^{4}$ et, chez les Papago, le succès croissant de la manifestation qui a fait passer le groupe de six pèlerins en 
1997 à 198 en 2003. Dans les deux cas, il existe un responsable du pèlerinage, du chant, des pèlerins marchant en procession et un porteur d'eau. Cette dernière charge est indispensable, car le voyage dure entre 5 et 6 jours avec une température qui peut atteindre $40^{\circ} \mathrm{C}$ à l'ombre.

\begin{tabular}{|l|l|l|l|}
\hline \multicolumn{1}{|c|}{ Pèlerinage } & \multicolumn{1}{|c|}{$\begin{array}{c}\text { Lien avec des } \\
\text { institutions }\end{array}$} & \multicolumn{1}{c|}{ Charges } & \multicolumn{1}{c|}{$\begin{array}{c}\text { Origine } \\
\text { des pèlerins }\end{array}$} \\
\hline $\begin{array}{l}\text { De Villa de Cos à } \\
\text { Real de Catorce, San } \\
\text { Luis Potosi }\end{array}$ & Église du sanctuaire & $\begin{array}{l}\text { Gardien majeur } \\
\text { Gardien second } \\
\text { Gardien du chant } \\
\text { Gardien de file } \\
\text { Porteur d'eau } \\
\text { Porteur d'étendard } \\
\text { Porteur du drapeau mexicain } \\
\text { Frère de la parole } \\
\text { Responsable de la reliquia } \\
\text { Danseurs }\end{array}$ & $\begin{array}{l}\text { Chef-lieu, hameaux } \\
\text { appartenant à Villa de } \\
\text { Cos et hameaux situés } \\
\text { sur le parcours }\end{array}$ \\
\hline $\begin{array}{l}\text { De Tubutama à } \\
\text { Magdalena de Kino, } \\
\text { Sonora }\end{array}$ & $\begin{array}{l}\text { Chef-lieu de Tubu- } \\
\text { tama et Magdalena } \\
\text { de Kino } \\
\text { Fonctionnaires de la la } \\
\text { Réserve de Sells }\end{array}$ & $\begin{array}{l}\text { Chamane } \\
\text { Aide du chamane et Porteur } \\
\text { de l'image sainte de Pozo } \\
\text { Verde } \\
\text { Responsable de file (Chan- } \\
\text { teurs) }\end{array}$ & $\begin{array}{l}\text { Les Papago de la } \\
\text { Réserve de Sells se réu- } \\
\text { nissent à Tubutama } \\
\text { avec ceux de Caborca, } \\
\text { Sonora, pour y com- } \\
\text { mencer la marche }\end{array}$ \\
\hline
\end{tabular}

FIG. 2-Organisation religieuse.

Du côté des pèlerins de Villa de Cos, la marche sur des sentiers éloignés des pistes les oblige à transporter de l'eau et à se ravitailler aux croisements des routes. Les chants sont dirigés par le responsable du pèlerinage ; entonnés pendant tout le chemin, ils évoquent les miracles de l'image sainte et ses victoires face aux pécheurs. Le drapeau mexicain et l'étendard du pèlerinage métis rappellent le conflit du mouvement cristero, qui opposa l'Église catholique et le gouvernement mexicain en 1930 (Figure 3). À ce moment-là, les Cristeros étaient censés être les fidèles et les federales, les autorités mexicaines, les pécheurs. Si l'on distingue la provenance des pèlerins d'après les étendards portant le nom de chaque village, le pèlerinage est reconnu comme étant celui de la municipalité de Villa de Cos. Néanmoins, comme le chef-lieu possède le même nom que la municipalité tout entière, des conflits surgissent. En effet, les pèlerins venant de la ville se disputent l'origine du pèlerinage avec ceux de Sierra Hermosa, hameau où celui-ci puise son origine et dont le nom apparaît sur l'étendard principal de la procession. La reliquia ${ }^{5}$ est le nom donné au repas collectif offert par un pèlerin. Le responsable de la danse ${ }^{6}$ regroupe les jeunes se dirigeant, en dansant, vers les pèlerins qui arrivent au sanctuaire. Le responsable du pèlerinage, le celador mayor, organise avec les autorités religieuses du sanctuaire l'entrée à l'église et la bienvenue. Le « frère de la parole » célèbre un service religieux, récite le chapelet et intègre les nouveaux pèlerins pendant le voyage. 


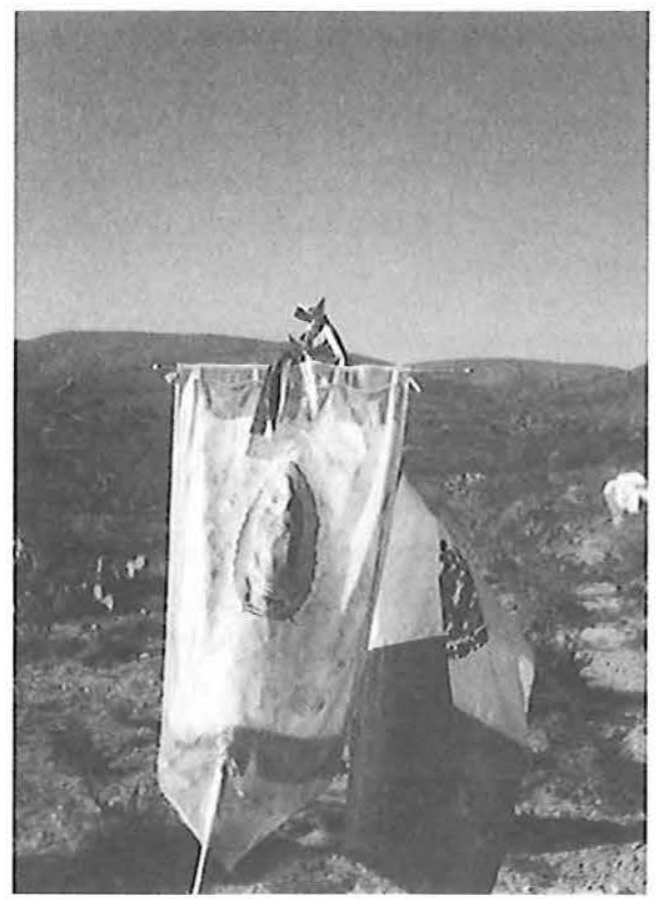

Fig. 3 - Le drapeau mexicain et l'étendard, symboles du pèlerinage de Villa de Cos, Zacatecas (cliché d'Alvarado Solís).

Du côté des pèlerins papago, le chamane doit bénéficier des services d'un assistant, lui-même versé dans la pratique chamanique. Ces charges rappellent l'initiation de naguère, lors du pèlerinage aux salines du Golfe de Californie. Chamane et aide effectuent tous deux les purifications ou limpias avec des bâtons à plumes, dans la fumée de la hediondilla (Larrea tridentata), une plante buissonnante du désert. Bâtons et images saintes sont portés par le chamane et son assistant, en tête de la procession des pèlerins. Des chants sont entonnés par des jeunes gens au lever du soleil, afin de placer le voyage sous de bons auspices, comme il était de coutume chez les Papago, le chant étant associé à la « magie d'un animal ", à savoir à sa faculté d'exaucer des vœux (Underhill 1940, pp. 55-57). Les grands-pères encourageaient leurs petits-fils à travailler dur afin de recevoir cette magie de l'animal. Tout au long du parcours, les Papago reçoivent l'assistance des autorités civiles des chefs-lieux traversés, en accord avec les autorités traditionnelles de la Réserve américaine de Sells. C'est grâce à cette organisation religieuse que peut être effectué ce périlleux voyage de plusieurs jours à travers le désert. 
L'organisation religieuse de Villa de Cos, centrée sur une idéologie manichéenne exprimant un combat entre les forces du bien et celles du mal, rappelle d'autres moments de l'histoire mexicaine. Les chants en l'honneur de l'image sainte et la lutte entre les danseurs évoquent des scènes de confrontation entre Indiens et Espagnols lors de la Conquête du Mexique. En revanche, chez les Papago, le chamane, l'initié et les chanteurs sont titulaires de charges relevant d'une organisation fort ancienne.

\section{Comment peut-on être pèlerin dans le désert?}

La route que suivent les fidèles de Villa de Cos n'est pas toujours la même : elle varie en fonction des conditions météorologiques et des recommandations des villageois. Chez les Papago, on marchait autrefois à travers champs, tandis qu'aujourd'hui on emprunte la route.

La carte reproduite (Figure 4) montre les lieux de départ des pèlerinages et les sanctuaires d'arrivée. La direction suivie est, dans les deux cas, d'ouest en est, soit vers le lever du soleil, qui est perçu, dans le monde indien, comme le lieu de la vie, tandis qu'il représente, dans le monde catholique, le lieu du Christ.

\section{Nommer le désert}

Les villages d'où partent les pèlerins, les déplacements effectués lors des pèlerinages et les sanctuaires s'inscrivent dans un espace non homogène : les plaines, la montagne et les ravins. Cependant, les pèlerins ne considèrent pas cet espace comme un " désert ». Seuls les Papago possèdent, dans leur langue, un mot qui désigne le désert (tohono). C'est aussi un terme utilisé comme ethnonyme par les Papago d'Arizona, Tohono O'otham (" gens du désert »), marquant d'ailleurs le rejet du nom attribué par les Espagnols à cette population, à savoir " Papago ». La plupart des pèlerins désignent cet espace sous le nom espagnol de monte qui peut signifier " espace sauvage » ou « espace non habité ». D'autres pèlerins le définissent comme un endroit vide et solitaire. Dans ce cas, ils utilisent le mot espagnol sólido, confondu en réalité avec solo, puisque l'on entend signifier que c'est un lieu sans personne et, de ce fait, qui permet d'être près de Dieu.

Ces espaces non habités sont craints, car ils abritent des espèces animales et végétales considérées comme dangereuses pour la santé des hommes. Ce danger oblige donc le pèlerin, qui se déplace pendant des jours à pied, à parcourir le désert en groupe et à ritualiser le déplacement en invoquant les images saintes pour assurer sa protection.

\section{Le départ}

Les pèlerins prêts à partir se retrouvent à la date et au lieu convenus. De Villa de Cos, ils se rendent à Sierra Hermosa où les villageois les attendent avec l'image sainte locale pour se diriger en procession à l'église, avec les autres pèlerins des hameaux participants. 


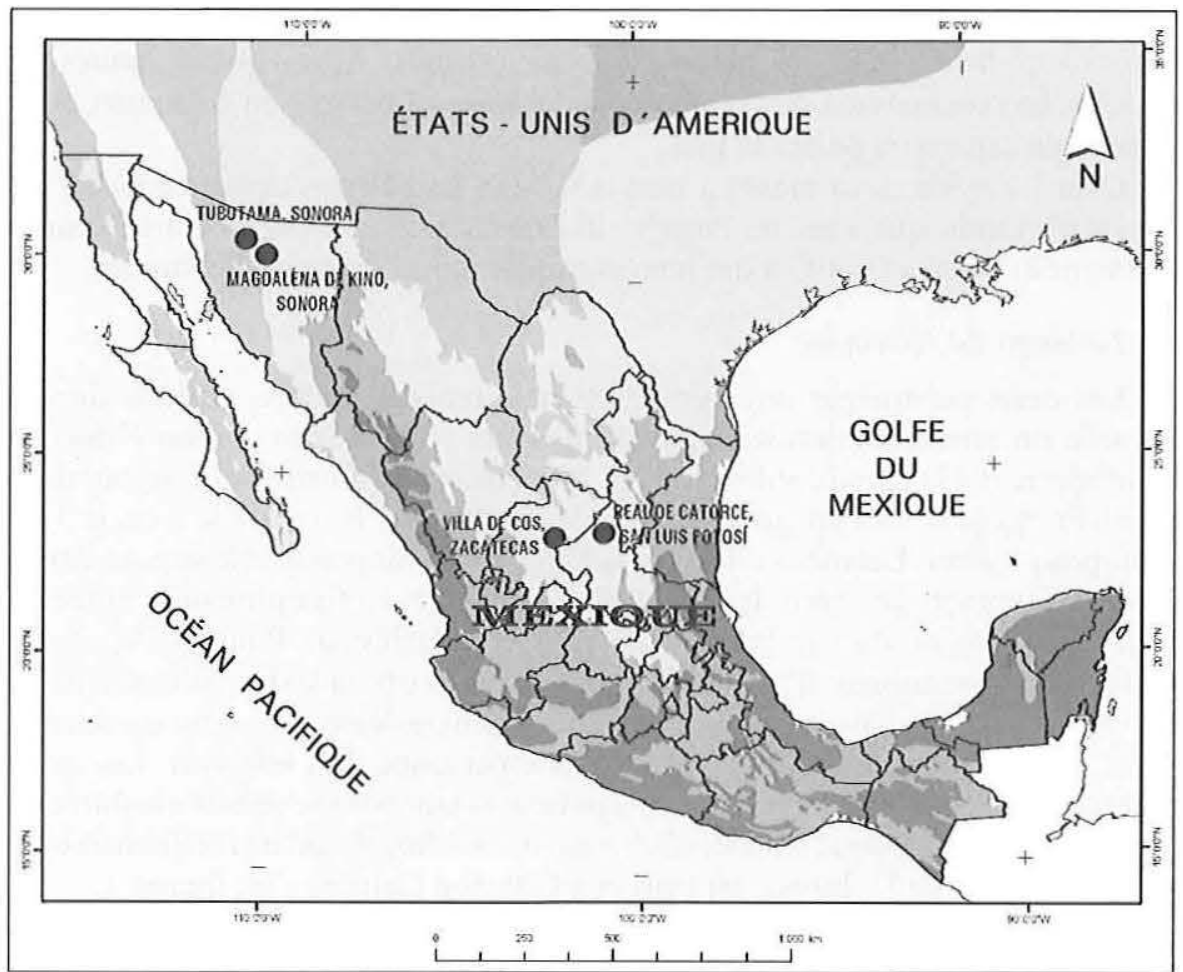

FIG. 4-Sanctuaires et villages de départ des pèlerinages des déserts sorense et chihuahuense (carte de Luis Olvera).

Papago et métis pratiquent un rite de départ. Les pèlerins métis l'effectuent à la chapelle de Sierra Hermosa, où le « frère de la parole » les bénit en passant l'étendard principal et le drapeau mexicain au-dessus de leurs têtes. Ces insignes sont aussi ceux que les nouveaux pèlerins doivent embrasser. À chaque hameau, des aspirants rejoignent le groupe des marcheurs et on les intègre de la même façon. À l'aube, commence la marche accompagnée de l' Ave Maria ${ }^{7}$ et les drapeaux reprennent leur place, au milieu des files processionnaires distribuées en fonction du sexe des pèlerins. Pendant tout le voyage, les femmes vont en tête; en pénétrant dans le territoire où se situe le sanctuaire, elles échangent leur place avec les hommes.

Le départ des Papago se déroule comme suit : rassemblés sur la place de Tubutama, ils reçoivent du chamane leurs bâtons de pèlerins. Le chamane les guide vers l'église pour y réciter un chapelet et toucher les images saintes avec ce bâton de façon à protéger les pénitents. Sur la place, le chamane, un fonctionnaire culturel de la réserve et une femme de Caborca adressent conseils et vœux aux marcheurs. La procession prend la route au coucher du soleil. Quelques pèlerins 
portent leurs affaires sur le dos, mais, en raison du poids, ils y renoncent au bout d'une demi-heure pour les placer dans les voitures. Après quatre heures de marche, les conducteurs des véhicules de la Réserve partent en éclaireurs pour trouver un espace où passer la nuit.

Chez les métis, pour mener à bien le voyage, les pèlerins doivent toucher les étendards tandis que, chez les Papago, ils doivent porter le bâton octroyé par le chamane et placé au contact des images saintes dans l'église de Tubutama.

\section{Le temps du pèlerinage}

Les deux pèlerinages ont lieu pendant le mois d'octobre, période durant laquelle on attend les dernières pluies, appelées el cordonazo de San Francisco (littéralement « le grand cordon de saint François »). Le 4 octobre est le jour de la Saint-François et les Papago arrivent à Magdalena de Kino dès le 2 ou le 3 du mois pour le fêter. Les métis célèbrent la Saint-François pendant le voyage, car ils arrivent au sanctuaire après la date festive. Papago et métis estiment la durée du voyage en fonction du nombre de nuits passées à l'extérieur. Pour les Papago, le chiffre 4 est un marqueur d'activité et l'unité de mesure pour la durée du pèlerinage correspond au fait d'avoir " passé quatre nuits dans le désert » (cuatro quedadas). C'est aussi le nombre de pèlerinages qu'une personne doit effectuer. Les métis mentionnent le nom des hameaux où ils passent la nuit pour se référer à la durée du pèlerinage. Ainsi, comme le dit le celadormayor : " à Bozalc'est une, à Victoria c'est deux, à Tanque de Dolores c'est trois et à Estación Catorce c'est quatre ».

Chez les Papago, d'autres moments importants sont la rencontre des animaux et/ou la découverte de leurs traces. Quand cela se produit, le chamane doit arrêter la marche pour " purifier » les espaces et les pèlerins. Chez les métis, on entonne des Ave Maria pendant toute la journée pour protéger les fidèles : ils sont censés ouvrir le temps de repos et d'occupation de l'espace. Les limpias papago et les séries d'Ave Maria prennent au moins trois quart d'heure chacune et sont effectuées à des intervalles réguliers tout au long de la journée. Chez les Papago, le déplacement se fait de jour et de nuit, tandis que les métis se déplacent seulement au petit matin et pendant la journée (Figure 5).

\begin{tabular}{|c|c|c|c|c|c|c|c|}
\hline Pèlerinage & $\begin{array}{c}\text { Temps } \\
\text { de la } \\
\text { marche }\end{array}$ & $\begin{array}{c}\text { Façon } \\
\text { de se } \\
\text { déplacer }\end{array}$ & Durée & $\begin{array}{c}\text { Temps } \\
\text { passé au } \\
\text { sanctuaire }\end{array}$ & $\begin{array}{c}\begin{array}{c}\text { Dates } \\
\text { du } \\
\text { pèlerinage }\end{array} \\
\end{array}$ & Saison & $\begin{array}{c}\text { Date } \\
\text { de la fète }\end{array}$ \\
\hline $\begin{array}{l}\text { De Villa de Cos } \\
\text { à Real de } \\
\text { Catorce, San } \\
\text { Luis Potosi }\end{array}$ & $\begin{array}{l}\text { Au petit } \\
\text { matin et } \\
\text { diurne }\end{array}$ & À pied & 4 nuits & $\begin{array}{l}\text { Une demi- } \\
\text { journée }\end{array}$ & $\begin{array}{l}\text { Du } 1^{\text {er }} \text { au } \\
5 \text { octobre }\end{array}$ & $\begin{array}{l}\text { Au début de la } \\
\text { saison sèche } \\
\text { Cordonazo de } \\
\text { San Francisco }\end{array}$ & 4 octobre \\
\hline $\begin{array}{l}\text { De Tubutama à } \\
\text { Magdalena de } \\
\text { Kino, Sonora }\end{array}$ & $\begin{array}{l}\text { Nocturne- } \\
\text { diurne }\end{array}$ & À pied & 5 nuits & 3 à 5 jours & $\begin{array}{l}\text { Du } 28 \text { sep- } \\
\text { tembre au } \\
3 \text { octobre }\end{array}$ & $\begin{array}{l}\text { Au début de la } \\
\text { saison sèche } \\
\text { Cordonazo de } \\
\text { San Francisco }\end{array}$ & 4 octobre \\
\hline
\end{tabular}

FIG. 5 - Quelques repères temporels des deux pèlerinages. 
Les données que l'on peut recueillir sur les durées et les distances témoignent de formes très spécifiques de l'espace/temps dans ce contexte. De fait, le déroulement du pèlerinage est plus important que son début et son arrivée. Le voyage rituel peut se faire seulement si l'on consacre toute la journée à des activités rituelles comme les purifications et les Ave Maria. On remarquera aussi le court séjour des pèlerins métis au sanctuaire par rapport au long voyage pèlerin car, comme l'explique une femme, " on vient juste voir saint François » (sólo venimos a ver a San Francisco). Chez les Papago, il existe une étroite équivalence entre le temps du voyage et le temps du séjour au sanctuaire. Malgré cette différence entre les deux pélerinages, l'intense activité rituelle des deux parcours dénote une même idée des durées et des distances. On notera enfin une correspondance entre les dates des deux voyages rituels : la fin de la saison des pluies, laquelle laisse ses traces sur le paysage, notamment sous la forme d'étangs adéquats pour des activités agricoles et de pâturage.

\section{L'espace du pèlerinage et les activités rituelles}

Les pèlerins de Villa de Cos passent par divers parajes ${ }^{8}$ et par les hameaux de Bozal, Victoria, Cardoncita, Tanque de Dolores, Rancho, Estación Catorce, Carretas, Catorcito et Real de Catorce (Figure 6). Dans les parajes, sont pris les repas. Quant aux hameaux, on y passe tous les ans et de nuit : ce sont des haltes. Les autres points signalés sur la carte sont les hameaux d'origine des pèlerins.

Les pèlerins papago passent, eux, par des parajes, ainsi que par des ponts, une rivière ' , une chapelle, Las Cebollas et l'entrée d'une mine, pour arriver finalement à Magdalena de Kino. Ces endroits sont indiqués, en chemin, par le chamane. Il s'agit essentiellement de lieux de partage de nourriture, de repos et de sommeil nocturne. Las Cebollas est le seul hameau habité du parcours (Figure 7).

Ces données laissent entrevoir l'importance des noms des lieux où l'on passe les nuits du voyage et le nombre de nuits que l'on doit y passer. Chez les Papago, on identifie la grande surface non habitée et le chiffre rituel 4. Chez les métis, les noms des endroits habités indiquent les réseaux de solidarité et de parenté rituelle qu'ils ont construits avec la population locale.

D'autres endroits, signalés par les pèlerins, motivent des actions rituelles et sont désignés comme des surfaces vides. Pour les pèlerins de Villa de Cos, il s'agit du territoire entre Victoria et Cardoncita, chemin considéré comme très diffícile à cause de la petite montagne qu'il faut escalader pour arriver ensuite dans une vallée de cactus. Cette difficulté est caractéristique de l'espace nommé monte. Il en existe d'autres liées à la topographie, c'est le cas de la "Côte du repenti » (Cuesta del Arrepentido): il s'agit d'une montée raide qu'il faut gravir de Carretas jusqu'à Real de Catorce. Ces lieux sont propices aux pratiques rituelles. L'Ave Maria ouvre et clôt la marche de la journée (Figure 8); il marque aussi des moments de repos et de repas. Les chants adressés à l'image sainte rendent le 


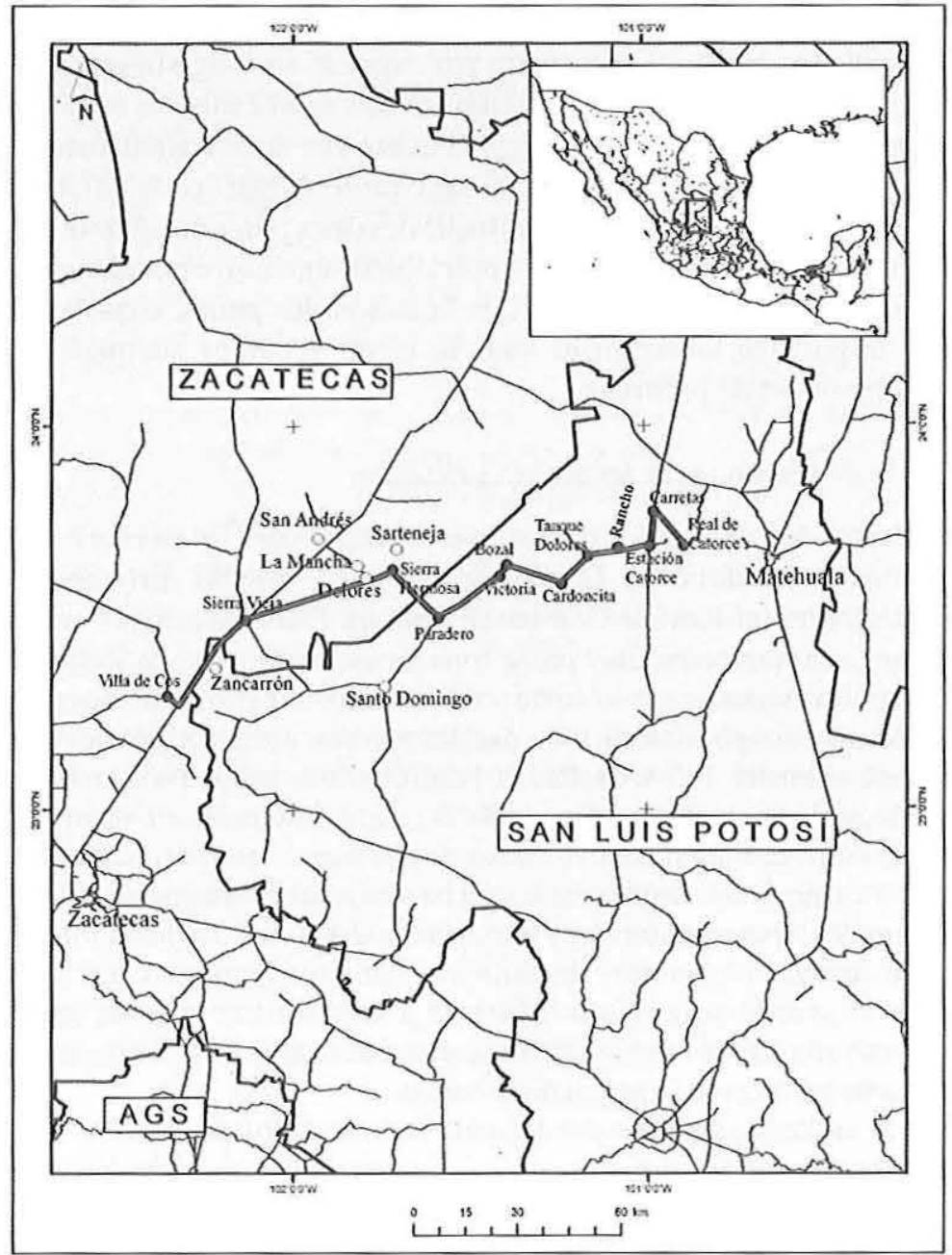

FIG. 6 - Route du pèlerinage métis de Villa de Cos, Zacatecas à Real de Catorce, San Luis Potosi (carte de Luis Olvera). 


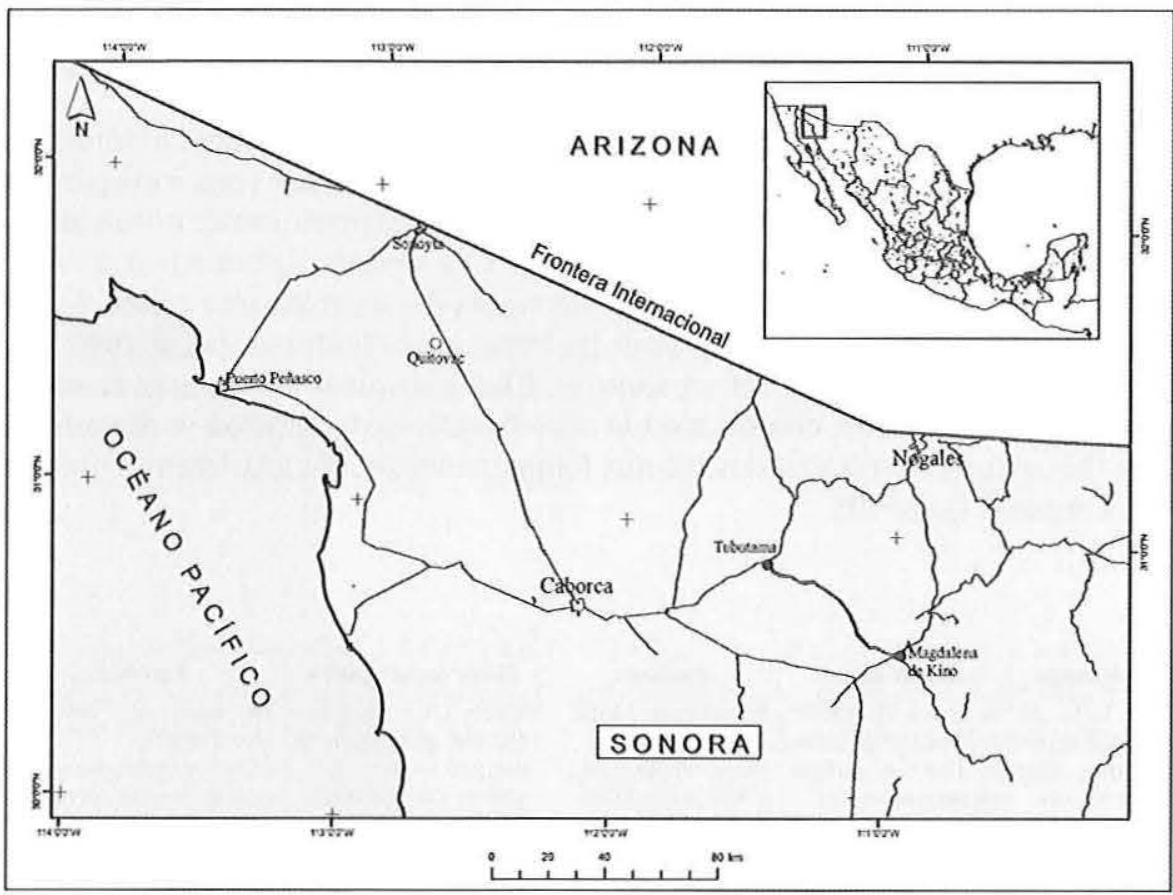

FIG. 7 - Route du pèlerinage papago à Magdalena de Kino, Sonora (carte de Luis Olvera).

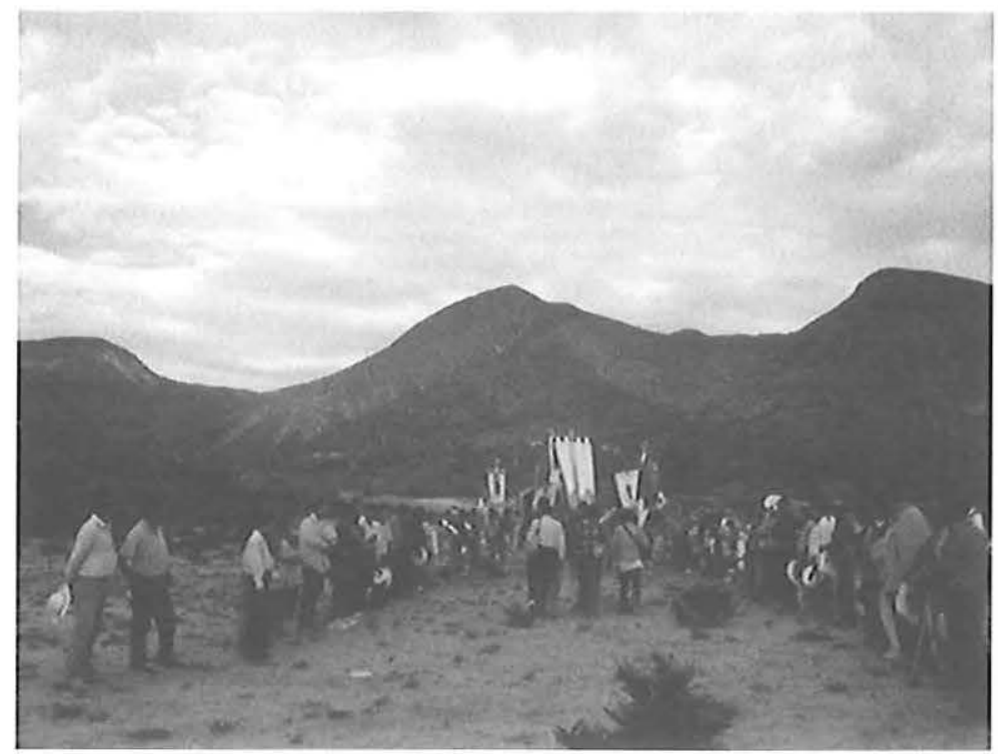

FIG. 8 - Ave Maria du pèlerinage métis (cliché d'Alvarado Solis). 
monte plus accessible et le chemin moins fatigant. Ils facilitent le voyage et soulagent la fatigue.

Pendant le trajet des Papago, le chamane leur montre des traces d'animaux, des plumes d'oiseaux, des animaux morts et il leur signale des sons émis par des serpents. Avec ses bâtons à plumes, le chamane, par des pratiques de purification, construit des ponts imaginaires sur le chemin. Ces " ponts » permettent d'éviter que les pèlerins entrent en contact avec les traces des animaux car celles-ci sont dangereuses pour eux (Figure 9). Pour les Papago, ces pratiques permettent aux pèlerins de parcourir le désert en sécurité. Elles marquent aussi l'arrivée sur le territoire du sanctuaire, comme c'est le cas à l'entrée de la mine où se déroule un acte thérapeutique collectif destiné aux femmes enceintes et aux femmes qui ont leurs règles (Figure 10).

\begin{tabular}{|c|c|c|c|c|}
\hline Pèlerinage & Rite de sortie & Parcours & Lieux remarquables & Activités \\
\hline $\begin{array}{l}\text { De Villa de } \\
\text { Cosà Real de } \\
\text { Catorce, San } \\
\text { Luis Potosi }\end{array}$ & $\begin{array}{l}\text { À Sierra Hermosa, } \\
\text { on bénit les pèlerins } \\
\text { et l'on en intègre } \\
\text { de nouveaux qui } \\
\text { n'étaient pas pré- } \\
\text { sents l'année précé- } \\
\text { dente }\end{array}$ & $\begin{array}{l}\text { En véhicule : Villa } \\
\text { de Cos-La Man- } \\
\text { cha-Dolores-entrée } \\
\text { à Sierra Hermosa. } \\
\text { Ä Pied : Sierra } \\
\text { Hermosa-Paraje- } \\
\text { Bozal-Victoria- } \\
\text { Cardoncita-Tanque } \\
\text { de Dolores-Rancho- } \\
\text { Estación Catorce- } \\
\text { Carretas-Catorcito- } \\
\text { Real de Catorce }\end{array}$ & $\begin{array}{l}\text { Victoria-Cardoncita: } \\
\text { chemin plus diflicile } \\
\text { nommé monte "sau- } \\
\text { vage», "non habité». } \\
\text { Carretas-Real de } \\
\text { Catorce: "Montée } \\
\text { du repenti ", montée } \\
\text { très abrupte }\end{array}$ & $\begin{array}{l}\text { De nouveaux pèlerins } \\
\text { sont intégrés. } \\
\text { L'Ave Mfaria ouvre et } \\
\text { clôt la marche pendant } \\
\text { la journée. Il marque le } \\
\text { début du repos, les repas, } \\
\text { sécurise l'espace. } \\
\text { Chants adressés à } \\
\text { l'image sainte: pour ren- } \\
\text { dre moins difficile le che- } \\
\text { min, inviter les pécheurs } \\
\text { à s'intégrer au pèle- } \\
\text { rinage, prendre congé } \\
\text { de la population des } \\
\text { hameaux. } \\
\text { Récitations de chapclets. } \\
\text { Un grand cercle est } \\
\text { eflectué à l'entrée du ter- } \\
\text { ritoire du sanctuaire. } \\
\text { On passe les nuits dans } \\
\text { des hameaux }\end{array}$ \\
\hline $\begin{array}{l}\text { De Tubuta- } \\
\text { ma à Magda- } \\
\text { lena de Kino, } \\
\text { Sonora }\end{array}$ & $\begin{array}{l}\text { Veillée à Pozo } \\
\text { Verde. On dit des } \\
\text { chapelets. } \\
\text { À Tubutama : on } \\
\text { récite un chapelet ; } \\
\text { le chamane octroye } \\
\text { aux pèlerins leurs } \\
\text { bâtons, on touche } \\
\text { les images saintes, } \\
\text { on écoute ses con- } \\
\text { seils }\end{array}$ & $\begin{array}{l}\text { Pozo Verde- } \\
\text { Tubutama-Paraje- } \\
\text { Arroyo-Capilla- } \\
\text { Puente-Cebollas- } \\
\text { Arroyo-Puente- } \\
\text { Paraje-Entrée d'une } \\
\text { mine-Magdalena de } \\
\text { Kino }\end{array}$ & $\begin{array}{l}\text { Pendant le trajet, le } \\
\text { chamane signale des } \\
\text { traces d'animaux, des } \\
\text { plumes d'oiseaux, } \\
\text { des animaux morts et } \\
\text { des sons émis par des } \\
\text { serpents }\end{array}$ & $\begin{array}{l}\text { Chants au lever du soleil } \\
\text { Purification des pèlerins } \\
\text { au zénith. } \\
\text { Repos. } \\
\text { Purification collective à } \\
\text { l'entrée du territoire du } \\
\text { sanctuaire. } \\
\text { Nuits passées dans le } \\
\text { désert }\end{array}$ \\
\hline
\end{tabular}

FIG. 9 - Lieux importants durant le voyage. 


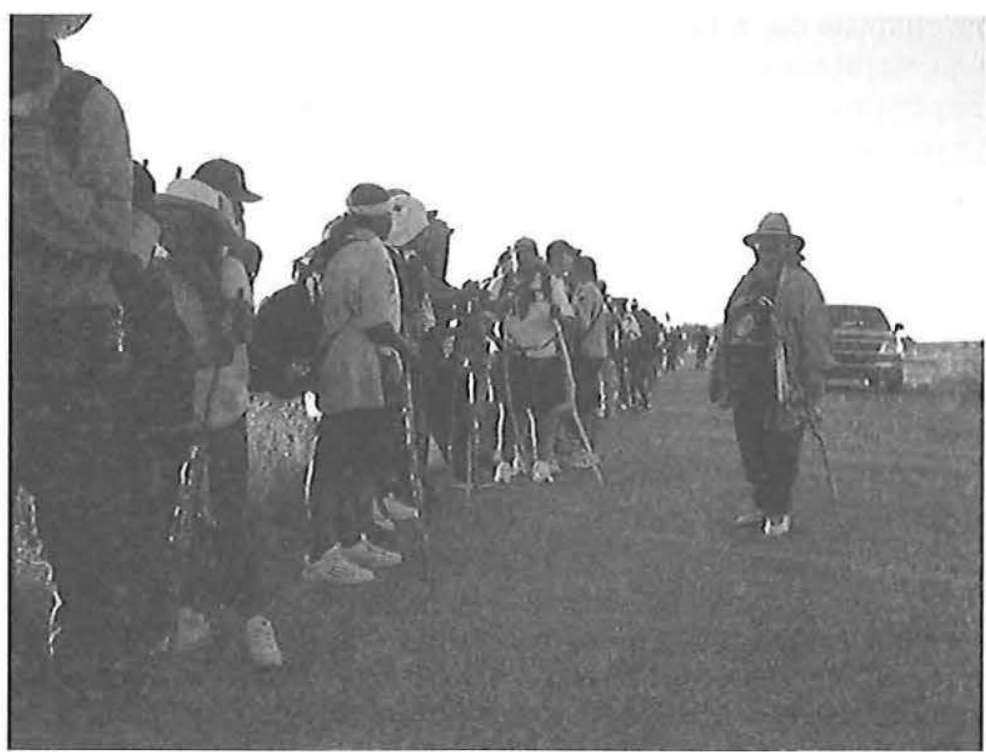

FIG. 10 - Pèlerinage papago à Magdalena de Kino, Sonora (cliché d'Alvarado Solís).

Lors du pèlerinage métis, les activités accomplies tout au long du voyage sont des prières, des chants, des Ave Maria, des repas, des moments de repos et d'intégration des nouveaux pèlerins, de partage avec la population des hameaux qui hébergent les pèlerins. Pour les Papago, les activités majeures sont les chants au lever du soleil, les limpias des pèlerins au zénith, les repas, les moments de repos, la visite aux images saintes de la chapelle qui se trouve sur le chemin, l'établissement de ponts symboliques et la recherche d'un espace pour passer la nuit.

L'entrée sur le territoire du sanctuaire est signalée dans les deux pèlerinages. Souvent la fatigue extrême causée par un chemin interminable est un signe qui annonce que l'on approche de l'entrée. Pour les Papago, on a déjà signalé l'acte collectif de purification, avec un traitement spécial pour certaines femmes. Pour les pèlerins métis à Real de Catorce, cet acte de purification a lieu sur la Cuesta del Arrepentido, montée diffĩcile, effectuée dans un esprit de repentance des péchés.

Ces données indiquent comment l'espace qualifié de "vide» est un lieu dangereux. Chez les Papago, le danger est lié au corps en crise à cause du désert et des états corporels féminins. Chez les métis, il est associé aux animaux et aux plantes, mais aussi à la fatigue du corps, assimilée à un sacrifice.

Dans le sanctuaire de Real de Catorce, les lieux les plus importants sont l'église, le cimetière, la place et La Cuesta del Arrepentido (Figure 11). Des activités s'y déroulent durant la fête de la Saint-François. Au cimetière, il y 
avait une chapelle où, à l'origine, se trouvait l'image du fondateur des franciscains, laquelle fut transportée dans l'église principale. Le jour de la fête, les fidèles se dirigent en procession vers le cimetière pour assister à la messe. Le marché fait partie du parcours de la procession, ainsi que le centre du village où se trouve une grande quantité de commerces.

\begin{tabular}{|l|l|}
\hline \multicolumn{1}{|c|}{ Pèlerinage } & \multicolumn{1}{c|}{ Lieux du sanctuaire } \\
\hline $\begin{array}{l}\text { De Villa de Cos à Real de } \\
\text { Catorce, San Luis Potosi }\end{array}$ & $\begin{array}{l}\text { Église, cimetière, place et Cuesta del } \\
\text { Arrepentido ou « Montée du repenti ». }\end{array}$ \\
\hline $\begin{array}{l}\text { De Tubutama à Magda- } \\
\text { lena de Kino, Sonora }\end{array}$ & Église, tombe du père Kino. \\
\hline
\end{tabular}

FIG. 11 - Lieux dans le sanctuaire.

À Magdalena de Kino, c'est à l'église avec l'autel de saint François-Xavier que l'on arrive en premier. La tombe du père Kino est visitée, puis l'on peut assister sur le parvis à des danses des Indiens yaqui, ou l'on se promène dans le centre de la ville, parsemé de nombreux commerces (Figure 12).

\begin{tabular}{|c|c|c|}
\hline Pèlerinage & Activités dans le sanctuaire & Caractéristiques du sanctuaire \\
\hline $\begin{array}{l}\text { De Villa de Cos à Real } \\
\text { de Catorce, San Luis } \\
\text { Potosi }\end{array}$ & $\begin{array}{l}\text { Les pèlerins en procession, séparés par } \\
\text { sexe, se préparent à entrer dans l'église, } \\
\text { à voir, toucher, chanter et dire au } \\
\text { revoir à l'image de saint François. } \\
\text { À l'entrée, les religieux donnent la } \\
\text { bienvenue aux pèlerins en annonçant } \\
\text { par haut-parleur qu'il s'agit de pèle- } \\
\text { rins à pied. } \\
\text { On prend le déjeuner, puis est effectué } \\
\text { le voyage de retour en vélicule }\end{array}$ & $\begin{array}{l}\text { Une grande foule, des commerces } \\
\text { d'objets religieux, de nourriture, de } \\
\text { vêtements et d'ustensiles de cuisine }\end{array}$ \\
\hline $\begin{array}{l}\text { De Tubutama à Mag- } \\
\text { dalena de Kino, So- } \\
\text { nora }\end{array}$ & $\begin{array}{l}\text { Les pèlerins entrent dans l'église de } \\
\text { la même façon qu'ils sont arrivés } \\
\text { au sanctuaire. On voit et on touche } \\
\text { l'image sainte, tandis que les chan- } \\
\text { teurs chantent en langue o'otham. On } \\
\text { s'installe à l'endroit désigné pour cela. } \\
\text { On regarde les danses du cerf et des } \\
\text { Matachines des Indiens yaqui. } \\
\text { On se promène dans le sanctuaire, on } \\
\text { visite le bar o'otham et on achète des } \\
\text { objets. } \\
\text { Le retour se fait en véhicule }\end{array}$ & $\begin{array}{l}\text { Il y a foule, il existe de nombreux } \\
\text { commerces d'objets religieux, de } \\
\text { nourriture, de vêtements et d'usten- } \\
\text { siles de cuisine. Au Centre de visite } \\
\text { o'otham, se trouvent un espace } \\
\text { médical et un espace pour se nourrir. } \\
\text { Au bar o'otham, de la musique des } \\
\text { groupes o'otham, de la danse et des } \\
\text { boissons alcoolisées }\end{array}$ \\
\hline
\end{tabular}

FIG. 12 - Principales activités dans le sanctuaire.

Les pèlerins arrivent exténués et, devant l'image sainte, les émotions affleurent : les larmes et la joie se mêlent. Même au moment de prendre congé, les pleurs ne semblent pas se tarir. L'accomplissement de l'objectif des pèlerins, à savoir toucher l'image miraculeuse, favorise l'expression des sentiments.

Dès leur arrivée, les pèlerins se placent devant l'image sainte, puis ils effectuent le voyage de retour immédiatement après. Les Papago restent pour se divertir et 
achever le pèlerinage en portant les trois rubans dont les couleurs symbolisent celles du monde papago. C'est le chamane qui donne ces rubans: le rouge symbolise le sang, le vert, la terre et le bleu, la mer et le ciel. Le retour se fait individuellement. Chaque pèlerin a déjà constitué, par avance, les réseaux dont il aura besoin.

\section{Le corps}

Dans le pèlerinage métis, le voyage constitue un sacrifice du corps destiné à nourrir l'âme. La souffrance des pèlerins correspond à la dette à payer pour être près du Christ. Le pèlerinage est une façon d'obtenir des « indulgences », c'està-dire que les péchés soient pardonnés et que l'accès au paradis soit facilité. C'est un voyage à effet " posthume». Dans ce rituel, on perçoit l'usage des images bibliques transmises par les missionnaires pendant la colonisation (Valdés et Alvarado 2007).

Pour les Papago, le pèlerinage est un rite de passage qu'il faut effectuer quatre fois dans sa vie comme c'était l'usage pour l'initiation chamanique en allant aux salines du Golfe de Californie. Le désert, les animaux et les plantes font partie du corps de Notre Mère, l'ancêtre féminin Ho'ók, ogresse du monde des origines, considéré comme dangeureux et contaminant. Selon les pèlerins papago, saint François appartient aussi à ce monde, tout comme les femmes mentruées ou enceintes. C'est pourquoi le chamane doit purifier le corps de ces dernières.

Pour les pèlerins métis, le pèlerinage est un rite de passage au-delà de la mort. Il représente aussi un espace maîtrisé par les femmes qui marchent en tête des processions. Pour les Papago, c'est un voyage initiatique à l'instar de ceux qu'ils effectuaient autrefois pour la chasse, la collecte du sel et l'initiation chamanique. Il permet de prévenir tout contact avec les menaces du désert, des animaux et des qualités dangereuses, qui se trouvent par extension dans le monde papago et décrites sous la catégorie de ka:cim « objet dangereux », liée aux « chemins » et à la « force » (Bahr et al. 1974, pp. 19-21).

L'espace des pèlerins, métis et Indiens, montre bien leur système de représentation du désert. L'appropriation de ce dernier suit la logique du repérage des endroits aux moments où le pèlerin s'expose aux propriétés dangereuses du désert. Il n'existe pas de lieux précis à visiter, déterminés à l'avance, sauf pour les espaces habités. L'appropriation se fait pendant la marche, au cours du voyage. Chez les Papago, ressurgissent des croyances anciennes, dans un pèlerinage récent. Pour les métis, les données historiques sont insuffísantes pour guider notre interprétation, même si on peut supposer que leur conception du corps est influencée par les croyances issues du catholicisme.

Pour les Papago, l'image sainte possède des qualités du monde des origines : il s'agit de dangers existant à la fois dans le désert et dans les états corporels 
féminins liés à la reproduction. Lorsque le pèlerin entre en contact avec des objets ou des êtres dangereux, une activité rituelle s'impose alors sur l'empreinte territoriale de ces contacts; cette activité permet véritablement de comprendre le mode d'appropriation papago du désert. Chez les métis, l'image sainte affirme son pouvoir, en exigeant du pèlerin le sacrifice de son corps comme l'intercession nécessaire devant Dieu. Dans ce cas-là, la dangerosité du désert associée au sacrifice du corps marque des lieux précis dans le voyage pèlerin. Dans les deux exemples, il s'agit de lieux qui ne laissent pas de traces visibles sur le paysage, mais leur existence renvoie au savoir, à la mémoire et au temps cyclique.

\section{CONCLUSION}

Les deux pèlerinages mettent en évidence la façon dont les Papago et les métis peuvent vivre dans un milieu hostile. Pour les Papago, il s'agit d'une invention récente qui passe par un spécialiste rituel, le chamane, intégrant une dévotion catholique à leur cosmogonie. Pour les métis, cette invention est articulée à un savoir ancien, se référant à la Conquête et la Cristiada. Il s'agit plus d'une répétition que d'une transformation des savoirs, ce qui renvoie au temps cyclique. Autrement dit, on est devant une tradition dont l'historicité relève d'une tension entre la mémorisation des instants de traditions inventées et la régulation constante de l'histoire des origines (Menget et Molinié 1993, pp. 9-19).

La construction spatiale du "désert pèlerin » est donc déterminée par les sensations corporelles. Celles-ci sont particulièrement en éveil aux endroits dangereux situés aux croisements entre le parcours du pèlerin et les traces laissés par certains animaux considérés comme contaminants. D'autres signes d'entités appartenant au monde des origines, par exemple un cactus aux épines crochues, sont autant de motifs pour procéder à des pratiques thérapeutiques hautement ritualisées. Ces points de rencontre constituent des sortes de balises, permettant aux pèlerins de réorienter leurs parcours dans une direction plus sûre vers le sanctuaire où ils se rendent, car c'est vers là que leurs ancêtres les guident grâce aux indices qu'ils laissent à ces endroits cruciaux du désert. Cette façon progressive et tâtonnante de trouver son chemin ne nous conduit-elle pas à une idée proche de la notion du labyrinthe, telle qu'elle est observée ailleurs dans le monde (Rosenstiehl 2002) ? L'enroulement et le déroulement du fil d'Ariane sont attestés dans le Nord-Ouest chez les Mexicanero (Alvarado 2004) à travers l'idée d'un fil qu'ils doivent lier et défaire rituellement pour assurer leur reproduction et la continuation des cycles. Chez les Huichol, un fil lie les objets rituels aux divinités pendant le pèlerinage à Wirikuta : il doit pouvoir se dérouler en évitant le danger de rester enroulé, pendant l'accomplissement du rituel (Geist 2005, pp. 86-88).

Ces métaphores du fil et du labyrinthe rappellent le mythe où le héros culturel des Papago, en affrontant les Apaches, construit un labyrinthe pour protéger le 
lieu des origines de leurs assauts (Aguilar 2004, p. 159). Cette image renvoie en même temps à la structure d'un " octadédale » (Radding 1995, p. 79) au centre duquel se situe idéalement la maison papago. Le recours à cette forme pour penser l'espace désertique est particulièrement astucieux car elle traduit le fait de se rapprocher du centre en s'éloignant immédiatement vers la périphérie.

Ces deux pèlerinages expriment ainsi l'idée d'un labyrinthe dont le centre à atteindre est le sanctuaire et lieu des origines : là où habitent les ancêtres. Il s'agit d'une construction de l'espace focalisée sur ces carrefours qui déclenchent les rouages du savoir et de la mémoire. Ainsi, dans un espace qui, à première vue, semble vide et sans forme, le chemin des pèlerins enroule le fil qui les lie à leurs ancêtres. La ritualisation de l'espace permet de le dérouler et le sacrifice du corps d'éviter de rester prisonnier dans le monde des défunts. Cette façon de parcourir le désert implique une notion du temps qui oblige le pèlerin à suivre les traces des ancêtres, enroulant et déroulant sans cesse le lien avec eux. Cette conception de l'espace/temps est si énigmatique et si propre à ces populations du Nord-Ouest mexicain qu'elle incite à méditer indéfiniment sur le sens des pèlerinages du Grand Nord mexicain. *

* Manuscrit reçu en janvier 2008, accepté pour publication en juin 2010.

\section{Notes}

Ce texte a été rédigé dans le cadre du projet « Pèlerinages du désert, une étude comparative » financé par le Programme de rapatriement 2002-2003 du Conseil national de science et technologie (CONACYT) et par le Centre de recherche et d'études supérieures en anthropologie sociale (CIESAS), Mexique.

1. Le désert mexicain, région aride couverte de buissons xérophiles, occupe un cinquième du pays. Le désert de Chihuahua s'étend sur deux États nord-américains (Nouveau Mexique et Texas) et dix États de la République mexicaine. Le désert de Sonora recouvre l'Arizona et la Californie aux États-Unis et les États de Sonora, Basse Californie et Sinaloa au Mexique. À l'ouest et à l'est, les limites des deux déserts sont des chaînes de montagnes, la Sierra Madre Orientale et la Sierra Madre Occidentale, au sud les États de San Luis Potosi et Zacatecas (Wauer 1992, pp. 17-18).

2. Il s'agit de pèlerinages chrétiens ; celui des Papago relève d'un catholicisme indianisé.

3. Les problèmes d'alcoolisme du chamane ont motivé sa décision de partir en pèlerinage à Magdalena de Kino pour obtenir sa guérison.

4. Dès le départ et dans chaque hameau du parcours, de nouveaux pèlerins s'intègrent au groupe en touchant l'étendard et le drapeau mexicain.

5. Littéralement « relique », fragment du corps d'un saint ou d'un objet dont le culte est autorisé par l'Église catholique.

6. La danza, aussi connue comme " danse de la Conquête ", rappelle ce moment de l'histoire du Mexique. Elle est exécutée par des jeunes gens dont une fille appelée Malinche.

7. Le « Je vous salue Marie ».

8. Terme espagnol désignant un « endroit ", sous-entendu ici " pour se reposer ou pour manger ».

9. En espagnol arroyo. Au nord du Mexique, arroyo désigne le lit d'un cours d'eau qui peut avoir les dimensions d'un fleuve, même s'il n'est plein que pendant la saison des pluies. 


\section{RÉFÉRENCES CITÉES}

Aguilar Alejandro

2004 «Los símbolos del desierto. Territorialidad y sitios sagrados entre los o'odham (pimas y pápagos) ", in Alicia Barabas (éd.), Diálogos con el territorio. Simbolizaciones sobre el espacio en las culturas indigenas de México, vol. III, Instituto Nacional de Antropología e Historia, coll. «Etnografía de los pueblos indígenas de México», Mexico, pp. 150-173.

Alvarado Solis Neyra Patricia

2004 Titailpí... timokotonal. Atar la vida, trozar la muerte, el sistema ritual de los mexicaneros de Durango, Universidad Michoacana de San Nicolás de Hidalgo/Exconvento de Tiripetío, Morelia, Michoacán.

2007 «Los avatares de la memoria, pápagos (tohono o’otham) peregrinos hacia Magdalena de Kino ", in Miguel Olmos (éd.), Antropología de la Frontera, Colegio de la Frontera Norte-Tijuana/Editorial Porrua, Mexico, pp. 357-373.

Bahr Donald, Juan Smith, William Smith, Allison Hayden et Julian Hayden

1974 The short swift time of gods on earth. The Hohokam cronicles, University of California Press, Berkeley/Los Angeles/Londres.

Cramaussel Chantal et Salvador Álvarez

1994 "La peregrinación de San José del Tizonazo, Durango », in Carlos Garma Navarro y Roberto Shadow (éds), Las peregrinaciones religiosas: una aproximación, UAM, Mexico, pp. 169-212.

Del Moral González Paulina

2006 Peregrinación y culto al señor de Mapimi, ritualidad y religión en el Cañón de Jimulco, mémoire de maîtrise en anthropologie sociale, ENAH-CIESAS, Chihuahua.

GaLinier Jacques

1991 «From Montezuma to San Francisco : the wigita ritual in Pápago (Tohono o'otham) religion ", Journal of the Southwest, 33 (4), pp. 486-533.

GeIsT Ingrid

2005 Liminaridad, tiempo y significación : prácticas rituales en la Sierra Madre Occidental, Instituto Nacional de Antropología e Historia, coll. « científica ", Mexico.

GutiéRrez Arturo

2002 La peregrinación a Virikuta, Instituto Nacional de Antropología e Historia, coll. " Etnografía de los pueblos indígenas de México », Universidad de Guadalajara, Mexico.

Howart Sam et Enrique Lamadrid

1999 Pilgrimage to Chimayó, contemporary portrait of living tradition, Museum of New Mexico Press, Santa Fe. 
Menget Patrick et Antoinette Molinié

1993 "Introduction », in Aurore Becquelin et Antoinette Molinié (éds), Mémoire de la tradition, Société d'ethnologie, Nanterre, pp. 9-19.

\section{Monroy Patricia}

1995 Tila, las raices históricas de su importancia como centro de peregrinación en Chiapas, mémoire de licence en histoire, UNAM-FFYL, Mexico.

RADDING Cynthia

1995 "Entre el desierto y la sierra : las naciones o'dham y tegüima de Sonora, 1530-1840 », in Rabiela Teresa Rojas et Mario Humberto Ruz (éds), Historia de los pueblos indigenas de México, Instituto Nacional Indigenista/Centro de Investigaciones y Estudios en Antropología Social, Mexico.

\section{Reyna Alviso Raymundo}

2005 La Milicia de San Miguel, redes sociales en una práctica ritual de San Luis Potosí y Guanajuato, mémoire de maîtrise en anthropologie sociale, El Colegio de San Luis A. C., San Luis Potosi.

\section{ROSENSTIEHL Pierre}

2002 "Labyrinthe ", in Sylvain Auroux (éd.), Les notions philosophiques. Dictionnaire, encyclopédie philosophique universelle, tome 1, Presses Universitaires de France, Paris, pp. 1425-1432.

\section{SHADOw Robert et María Rodríguez SHADOw}

2000 El pueblo del Señor : las fiestas y peregrinaciones de Chalma, Universidad del Estado de México, col. « Historia » 22, Toluca.

UNDERHILL Ruth

1940 The Pápago Indians of Arizona and their relatives the Pima, Bureau of Indians affairs, Education division, Sherman pamphlets $\mathrm{n}^{\circ} 3$, Washington.

Valdés Carlos et Neyra Alvarado

2007 «El uso de la flora en el noreste mexicano, aproximaciones histórica y contemporánea ", in Andrés Fábregas Puig, Mario Alberto Nájera Espinoza et José Alfredo Ortiz Garza (éds), Diversidad cultural y sobrevivencia, la frontera chichimeca, una visión desde el siglo $x I x$, seminario permanente de estudios de la Gran Chichimeca, Universidad de Guadalajara, Universidad Autónoma de Zacatecas, Universidad Autónoma Aguascalientes, Universidad Intercultural de Chiapas, El Colegio de San Luis, El Colegio de Jalisco, El Colegio de Michoacán, Universidad Autónoma de Coahuila, Guadalajara, pp. 213-220.

Velasco Toro José

1997 Santuario y región del Cristo negro de Otatitlan, Universidad veracruzana, Instituto de Investigaciones histórico-sociales, Jalapa.

WAUER Roland

1992 A naturalist's México, Texas A y M University Press, College Station. 